\title{
The Characterizations of the Spherical Images of Timelike W-Curves in Minkowski Space-Time
}

\author{
Minkowski Uzay-Zamanda Timelike W-Eğrilerin Küresel \\ Göstergelerinin Karakterizasyonları \\ Yasin Ünlütürk1 (D), Süha Yılmaz $^{2 *}$ (D) Muradiye Çimdiker ${ }^{3}$ (D) \\ ${ }^{1,3}$ Kırklareli University, Kırklareli, TURKEY \\ 2 Dokuz Eylül University, İzmir, TURKEY \\ Sorumlu Yazar / Corresponding Author*: suha.yilmaz@deu.edu.tr
}

Geliş Tarihi /Received: 05.03.2019

Kabul Tarihi / Accepted: 29.07.2019

Araştırma Makalesi/Research Article DOI: $10.21205 /$ deufmd.2020226407

Atıfșekli/How to cite: ÜNLÜTÜRK, Y., YILMAZ, S., CIIMDIKER, M. (2020). The Characterizations of the Spherical Images of Timelike W-Curves in Minkowski Space-Time. DEUFMD, 22(64),59-66.

\begin{abstract}
We know that $W$-curve is a curve which has constant Frenet curvatures. In this study, firstly, we have investigated the principal normal and binormal spherical images of a timelike $W$-curve on pseudohyperbolic space $\mathbb{H}_{0}^{3}$ in Minkowski space-time $E_{1}^{4}$. Besides, the binormal spherical image of the timelike $W$-curve is a spacelike curve which lies on pseudohyperbolic space $\mathbb{H}_{0}^{3}$. Hence, we have obtained the Frenet-Serret invariants of the mentioned image curve in terms of the invariants of the timelike $\mathrm{W}$-curve in the same space. Finally, we have given some characterizations of the spherical image in the case of being helix for the timelike $W$-curve in Minkowski space-time $E_{1}^{4}$.

Keywords: Spherical Images, Timelike W-Curve, General Helix, CCR-Curves

Özet

$W$-eğrisinin sabit Frenet eğriliklerine sahip bir eğri olduğunu biliyoruz. Bu çalışmada, öncelikle, $E_{1}^{4}$ Minkowski uzay-zamanında, $\mathbb{H}_{0}^{3}$ pseudohiperbolik uzay üzerinde bir timelike $W$-eğrisinin asli normal ve binormal küresel göstergelerini araştırdık. Yanısıra, $\mathbb{H}_{0}^{3}$ pseudohiperbolik uzay üzerinde yatan timelike $W$-eğrisinin binormal küresel göstergesi spacelike bir eğridir. Bu nedenle, aynı uzayda, söz konusu görüntü eğrisinin Frenet-Serret değişmezlerini timelike $W$-eğrisinin değişmezleri cinsinden elde ettik. Son olarak, $E_{1}^{4}$ Minkowski uzay-zamanındaki timelike $W$-eğrisi için helis olması durumunda küresel göstergenin bazı karakterizasyonlarını verdik.

Anahtar Kelimeler: Küresel Göstergeler, Timelike W-Eğrileri, Genel Helis, CCR-Eğriler
\end{abstract}




\section{Introduction}

Lorentzian geometry helps to bridge the gap between modern differential geometry and the mathematical physics of general relativity by giving an invariant treatment of Lorentzian geometry. Nearly a century ago, Einstein's formulation of general relativity expressed in terms of Lorentzian geometry was attractive for geometricians who could penetrate suprisingly into cosmology (redshift, expanding universe, big bang)[1].

Despite its long history, the theory of curve is still one of the most interesting topics in differential geometry and it is still being studied by many mathematicians until now. A tetrad of mutually orthogonal unit vectors (called tangent, normal, binormal, trinormal) was defined and constructed at each point of a differentiable curve. The rates of change of these vectors along the curve define the curvatures of the curve in the four dimensional space. Spherical images of a regular curve in the Euclidean space are obtained by means of Frenet-Serret frame vector fields, so the mentioned topic is a well-known concept in differential geometry of the curves [2]. Also, these kind of curves were studied in four dimensional Euclidean and Lorentzian space $[3,4,5,6,7]$.

$\mathrm{W}$-curve is another curve among the prominent curves which have the constant Frenet curvature. All $W$-curves in Minkowski 3-space are completely classified by Walrave in [3]. Besides, in Minkowski space-time, the spacelike, timelike, null W-curves are studied [8,9].

In this study, we have investigated the principal normal and binormal spherical images of a timelike $W$-curve on pseudohyperbolic space $\mathbb{H}_{0}^{3}$ in Minkowski space-time $E_{1}^{4}$. The binormal spherical image of the timelike $\mathrm{W}$-curve is a spacelike curve which lies on pseudohyperbolic space $\mathbb{H}_{0}^{3}$. Hence, we have obtained the FrenetSerret invariants of the mentioned image curve in terms of the invariants of the timelike $W$-curve. Finally, we have given some characterizations of the spherical image in the case of being helix for the timelike $W$-curve in Minkowski space-time $E_{1}^{4}$.

\section{Material and Method}

Minkowski spacetime $E_{1}^{4}$ is a real vector space $R^{4}$ furnished with the standard indefinite flat metric $g$ defined by

$g=-d x_{1}+d x_{2}+d x_{3}+d x_{4}$,

where $\left(x_{1}, x_{2}, x_{3}, x_{4}\right)$ is a rectangular coordinate system in $E_{1}^{4}$ [1]. Since $g$ is an indefinite metric, recall that a vector $v \in E_{1}^{4}$ can have one of the three causal characters; it can be spacelike if $g(v, v)>0$ or $v=0$, timelike if $g(v, v)<0$ and null (lightlike) if $g(v, v)=0, v \neq 0$. Similarly, an arbitrary curve $\alpha=\alpha(s)$ in $E_{1}^{4}$ can be locally spacelike, timelike or null (lightlike), if all of its velocity vectors $\alpha^{\prime}(s)$ are spacelike, timelike or null (lightlike), recpectively. Also, the norm of the vector $v$ is given by

$$
\|v\|=\sqrt{|g(v, v)|} .
$$

The vector $v$ is a unit vector if $g(v, v)=\mp 1$. Vectors $v, w$ in $E_{1}^{4}$ are said to be orthogonal if $g(v, w)=0$ [10]. Let $u$ and $v$ be two spacelike vectors in $E_{1}^{4}$, then there is a unique real number $0 \leq \delta \leq \pi$, called the angle between $u$ and $v$, such that $g(u, v)=\|u\|\|v\| \cos \delta[11]$.

The pseudohyperbolic space with the center $m=\left(m_{1}, m_{2}, m_{3}, m_{4}\right) \in E_{1}^{4}$ and radius $r \in \mathbb{R}^{+}$in the spacetime $E_{1}^{4}$ is the hyperquadric

$$
\begin{aligned}
\mathbb{H}_{0}^{3}=\left\{a=\left(a_{1}, a_{2},\right.\right. & \left.a_{3}, a_{4}\right) \in E_{1}^{4} \\
& \left.\mid g(a-m, a-m)=-r^{2}\right\},
\end{aligned}
$$

with dimension 3 and index 0 [1].

Let $\varphi=\varphi(s)$ be a curve in $E_{1}^{4}$. If the tangent vector field of this curve forms a constant angle with a constant vector field $U$, then this curve is called a general helix. Recall that, if a regular curve has constant Frenet-Serret curvatures ratios in $E_{1}^{4}$, then it is called a ccr-curve $[12,13,14]$. Also, if these curvatures are non-zero constants, the curve is said to be $W$-curve (or helix) $[15,16,17]$.

Denote by $\left\{T(s), N(s), B_{1}(s), B_{2}(s)\right\}$ the moving Frenet-Serret frame along the curve $\varphi(s)$ in $E_{1}^{4}$. Then $T, N, B_{1}, B_{2}$ are, respectively, the tangent, the principal normal, the binormal (the first binormal) and the trinormal (the second binormal) vector fields. A spacelike or timelike curve $\varphi(s)$ is said to be parametrized by arclength function $s$, if $g\left(\varphi^{\prime}(s), \varphi^{\prime}(s)\right)= \pm 1$. 
DEU FMD 22(64), 59-66, 2020

Let $\varphi(s)$ be a timelike curve in $E_{1}^{4}$, parametrized by arc-length function $s$. Then the following Frenet-Serret equations are given in [3]:

$$
\left[\begin{array}{l}
T^{\prime} \\
N^{\prime} \\
B_{1}^{\prime} \\
B_{2}^{\prime}
\end{array}\right]=\left[\begin{array}{cccc}
0 & \kappa & 0 & 0 \\
\kappa & 0 & \tau & 0 \\
0 & -\tau & 0 & \sigma \\
0 & 0 & -\sigma & 0
\end{array}\right]\left[\begin{array}{c}
T \\
N \\
B_{1} \\
B_{2}
\end{array}\right],
$$

where $T, N, B_{1}, B_{2}$ are mutually orthogonal vectors satisfying equations

$g(T, T)=-1$,

$g(N, N)=g\left(B_{1}, B_{1}\right)=g\left(B_{2}, B_{2}\right)=1$,

and where $\kappa, \tau, \sigma$ are the first, second, and third curvatures of the curve $\varphi$, respectively.

In the same space, the authors expressed a characterization of spacelike curves lying on $\mathbb{H}_{0}^{3}$ by the following theorem:

Theorem 2.1. Let $\varphi(s)$ be an unit speed spacelike curve in $E_{1}^{4}$, with the spacelike vectors $\mathrm{N}, B_{1}$ and the curvatures $\kappa \neq 0, \tau \neq 0, \sigma \neq 0$ for each $s \in I \subset \mathbb{R}$. Then, the curve $\varphi$ lies on pseudohyperbolic space if and only if

$$
\frac{\sigma}{\tau} \frac{d \rho}{d s}=\frac{d}{d s}\left[\frac{1}{\sigma}\left(\rho \tau+\frac{d}{d s}\left(\frac{1}{\tau} \frac{d \rho}{d s}\right)\right)\right],
$$

where

$\left\{\frac{1}{\sigma}\left(\rho \tau+\frac{d}{d s}\left(\frac{1}{\tau} \frac{d \rho}{d s}\right)\right)\right\}^{2}>\rho^{2}+\left(\frac{1}{\tau} \frac{d \rho}{d s}\right)^{2}$

and $\rho=\frac{1}{\kappa}[15]$.

Definition 2.2. Let $a=\left(a_{1}, a_{2}, a_{3}, a_{4}\right), b=$ $\left(b_{1}, b_{2}, b_{3}, b_{4}\right)$ and $c=\left(c_{1}, c_{2}, c_{3}, c_{4}\right)$ be vectors in $E_{1}^{4}$. The vector product is defined by

$a \times b \times c=-\left|\begin{array}{cccc}-e_{1} & e_{2} & e_{3} & e_{4} \\ a_{1} & a_{2} & a_{3} & a_{4} \\ b_{1} & b_{2} & b_{3} & b_{4} \\ c_{1} & c_{2} & c_{3} & c_{4}\end{array}\right|$,

where $e_{1}, e_{2}, e_{3}, e_{4}$ are mutually orthogonal vectors (coordinate direction vectors) satisfying equations

$\begin{array}{ll}e_{1} \times e_{2} \times e_{3}=e_{4}, & e_{2} \times e_{3} \times e_{4}=e_{1}, \\ e_{3} \times e_{4} \times e_{1}=e_{2}, & e_{4} \times e_{1} \times e_{2}=-e_{3},\end{array}$

[5].
Theorem 2.3. Let $\varphi(\mathrm{s})$ be an arbitrary spacelike curve in $E_{1}^{4}$. The Frenet-Serret apparatus of the curve $\varphi$ can be written as follows:

$$
\begin{aligned}
T & =\frac{\varphi^{\prime}}{\left\|\varphi^{\prime}\right\|}, \\
\mathrm{N} & =\frac{\left\|\varphi^{\prime}\right\|^{2} \varphi^{\prime \prime}-\mathrm{g}\left(\varphi^{\prime}, \varphi^{\prime \prime}\right) \varphi^{\prime}}{\|\| \varphi^{\prime}\left\|^{2} \varphi^{\prime \prime}-\mathrm{g}\left(\varphi^{\prime}, \varphi^{\prime \prime}\right) \varphi^{\prime}\right\|}, \\
B_{1} & =\mu N \times T \times B_{2}, \\
B_{2} & =\mu \frac{T \times N \times \varphi^{\prime \prime \prime}}{\left\|T \times N \times \varphi^{\prime \prime \prime}\right\|},
\end{aligned}
$$

and

$$
\begin{aligned}
& \kappa=\frac{\|\| \varphi^{\prime}\left\|^{2} \varphi^{\prime \prime}-\mathrm{g}\left(\varphi^{\prime}, \varphi^{\prime \prime}\right) \varphi^{\prime}\right\|}{\left\|\varphi^{\prime}\right\|^{4}}, \\
& \tau=\frac{\left\|T \times N \times \varphi^{\prime \prime \prime}\right\|\left\|\varphi^{\prime}\right\|}{\|\| \varphi^{\prime}\left\|^{2} \varphi^{\prime \prime}-\mathrm{g}\left(\varphi^{\prime}, \varphi^{\prime \prime}\right) \varphi^{\prime}\right\|^{\prime}}, \\
& \sigma=\frac{g\left(\varphi^{I V}, B_{2}\right)}{\left\|T \times N \times \varphi^{\prime \prime \prime}\right\| \varphi^{\prime} \| \prime}
\end{aligned}
$$

where $\mu$ is taken -1 or 1 to make 1 the determinant of $\left\{T, N, B_{1}, B_{2}\right\}$ matrix [5].

\section{Results}

\subsection{The principal normal spherical image of a timelike $W$-curve in $E_{1}^{4}$}

In this section, we give the definition of the principal normal spherical image for the timelike $W$-curves in Minkowsk space-time $E_{1}^{4}$.

Definition 3.1. Let $\beta=\beta(s)$ be a unit speed timelike $W$-curve in Minkowski space-time $E_{1}^{4}$. If we translate the principal normal vector to the center 0 of the pseudohyperbolic space $\mathbb{H}_{0}^{3}$, we obtain a curve $\delta=\delta\left(s_{\delta}\right)$. This curve is called the principal normal spherical indicatrix or image of the curve $\beta$ in $E_{1}^{4}$.

Theorem 3.2. Let $\beta=\beta(s)$ be a unit speed timelike $W$-curve and $\delta=\delta\left(s_{\delta}\right)$ be its principal normal spherical image. Then,

i) $\delta=\delta\left(s_{\delta}\right)$ is a spacelike curve if the first and second curvatures of $\beta(s)$ satisfy the following

$$
\tau<\kappa<0, \quad 0<\kappa<\tau .
$$

ii)Frenet-Serret apparatus of the curve $\delta,\left\{T_{\delta}, N_{\delta}, B_{1 \delta}, B_{2 \delta}, \kappa_{\delta}, \tau_{\delta}, \sigma_{\delta}\right\}$ can be formed by the apparatus of the curve $\beta$. 
DEU FMD 22(64), 59-66, 2020

Proof. Let $\beta=\beta(s)$ be a unit speed timelike Wcurve and $\delta=\delta\left(s_{\delta}\right)$ be its principal normal spherical indicatrix. It can be written as

$$
\delta=N(s)
$$

Differentiating the equation (5) with respect to $s$, then we obtain

$$
\delta^{\prime}=\dot{\delta} \frac{d s_{\delta}}{d s}=\kappa T+\tau B_{1} .
$$

Here, we shall denote differentiation according to $s$ by a dash, and differentiation according to $s_{\delta}$ by a dot. Thus, we obtain the unit tangent vector of the principal normal spherical image curve $\delta$ as

$$
T_{\delta}=\frac{\kappa T+\tau B_{1}}{\sqrt{\tau^{2}-\kappa^{2}}}
$$

and

$$
\left\|\delta^{\prime}\right\|=\frac{d s_{\delta}}{d s}=\sqrt{\tau^{2}-\kappa^{2}} .
$$

The causal character of the principal normal spherical image curve $\delta$ is determined by the following inner product:

$$
g\left(\delta^{\prime}, \delta^{\prime}\right)=\tau^{2}-\kappa^{2} .
$$

From the expression (9), we will take the spherical image curve as spacelike one by assuming the conditions

$$
\tau<\kappa<0, \quad 0<\kappa<\tau .
$$

Considering the previous method and using the property of the curve to be $W$-curve, we form the following differentiations with respect to $s$ :

$$
\begin{aligned}
\delta^{\prime \prime}= & \left(\kappa^{2}-\tau^{2}\right) N+\tau \sigma B_{2}, \\
\delta^{\prime \prime \prime}= & \kappa\left(\kappa^{2}-\tau^{2}\right) T+\tau\left(\kappa^{2}-\tau^{2}\right. \\
& \left.-\sigma^{2}\right) B_{1} \\
\delta^{(I V)}= & \left(\left(\kappa^{2}-\tau^{2}\right)^{2}+\tau^{2} \sigma^{2}\right) N \\
& +\tau \sigma\left(\kappa^{2}-\tau^{2}-\sigma^{2}\right) B_{2} .
\end{aligned}
$$

By the expressions (2), we arrive at

$$
\begin{aligned}
\left\|\delta^{\prime}\right\|^{2} \delta^{\prime \prime}-g\left(\delta^{\prime}, \delta^{\prime \prime}\right) & \delta^{\prime} \\
= & -\left(\kappa^{2}-\tau^{2}\right)^{2} N \\
& +\tau \sigma\left(\tau^{2}-\kappa^{2}\right) B_{2}
\end{aligned}
$$

Then, we can write the principal normal vector of the spherical image curve $\delta$

$$
\begin{aligned}
N_{\delta}= & \frac{\kappa^{2}-\tau^{2}}{\sqrt{(\tau \sigma)^{2}+\left(\tau^{2}-\kappa^{2}\right)^{2}}} N \\
& +\frac{\tau \sigma}{\sqrt{(\tau \sigma)^{2}+\left(\tau^{2}-\kappa^{2}\right)^{2}}} \cdot B_{2}
\end{aligned}
$$

and the first curvature of the spherical image curve $\delta$ is obtained by

$$
\kappa_{\delta}=\frac{\sqrt{(\tau \sigma)^{2}+\left(\tau^{2}-\kappa^{2}\right)^{2}}}{\tau^{2}-\kappa^{2}} .
$$

Now, calculate the vector product

$U=T_{\delta} \times N_{\delta} \times \delta^{\prime \prime \prime}$, that is, we have the vector $U$ as

$$
U=\frac{-\kappa \tau \sigma^{2}}{\sqrt{\tau^{2}-\kappa^{2}}}\left(\begin{array}{c}
\frac{-\tau \sigma}{\sqrt{(\tau \sigma)^{2}+\left(\tau^{2}-\kappa^{2}\right)^{2}}} N \\
+\frac{\kappa^{2}-\tau^{2}}{\sqrt{(\tau \sigma)^{2}+\left(\tau^{2}-\kappa^{2}\right)^{2}}} B_{2}
\end{array}\right) .
$$

Hence, we obtain the trinormal (second binormal) vector field of the principal normal spherical image curve $\delta$ as follows:

$$
=\mu\left(\begin{array}{c}
\frac{\tau \sigma}{\sqrt{(\tau \sigma)^{2}+\left(\tau^{2}-\kappa^{2}\right)^{2}}} N \\
+\frac{\tau^{2}-\kappa^{2}}{\sqrt{(\tau \sigma)^{2}+\left(\tau^{2}-\kappa^{2}\right)^{2}}} B_{2}
\end{array}\right) .
$$

Taking the norm of both sides of the expressions (15) and (12) then the second curvature of the principal normal spherical image curve $\delta$ is

$$
\tau_{\delta}=\frac{-\kappa \tau \sigma^{2}}{\left(\tau^{2}-\kappa^{2}\right) \sqrt{(\tau \sigma)^{2}+\left(\tau^{2}-\kappa^{2}\right)^{2}}} .
$$

To obtain the binormal vector field of the principal normal spherical image curve $\delta$,we express $V=N_{\delta} \times T_{\delta} \times B_{2 \delta}$ as follows:

$$
V=-\frac{\tau}{\sqrt{\tau^{2}-\kappa^{2}}} T-\frac{\kappa}{\sqrt{\tau^{2}-\kappa^{2}}} B_{1} .
$$

From the expression (18), then we get the binormal vector of the principal normal spherical image curve $\delta$ 
DEU FMD 22(64), 59-66, 2020

$$
\begin{aligned}
& B_{1 \delta}=\mu\left(-\frac{\tau}{\sqrt{\tau^{2}-\kappa^{2}}} T\right. \\
&\left.-\frac{\kappa}{\sqrt{\tau^{2}-\kappa^{2}}} B_{1}\right)
\end{aligned}
$$

Using the equation (16), the third curvature is given by

$$
\sigma_{\delta}=\mu \frac{\kappa \sigma}{\sqrt{(\tau \sigma)^{2}+\left(\tau^{2}-\kappa^{2}\right)^{2}}} .
$$

Corollary 3.3. Frenet-Serret apparatus of the principal normal spherical image curve $\delta$ is an orthonormal frame of Minkowski space-time $E_{1}^{4}$.

Proof. It can be straightforwardly seen by using the equations (7), (13), (16), (19).

Corollary 3.4. Let $\beta=\beta(s)$ be a unit speed timelike $W$-curve and $\delta=\delta\left(s_{\delta}\right)$ be its principal normal spherical image. Then, the curve $\delta$ is also a helix.

Proof. Let $\beta=\beta(s)$ be a unit speed timelike $W$-curve. We know that the curvature functions are constants. Therefore, we know that the curvature functions of the principal normal spherical image $\delta\left(s_{\delta}\right)$ are constants by means of the equations (14), (17) and (20). Hence, the curve $\delta\left(s_{\delta}\right)$ becomes $W$-curve which is the special case of helix.

Theorem 3.5. Let $\beta=\beta(s)$ be a unit speed timelike $W-$ curve and $\delta=\delta\left(s_{\delta}\right)$ be its principal normal spherical image. If $\delta$ is a general helix, then its fixed direction $\Phi$ is composed

$$
\begin{aligned}
\Phi= & \left(-\frac{1}{2} x_{1} \kappa s^{2}-x_{2} \kappa s+x_{3}\right) T \\
& +\left(x_{1} s+x_{2}\right) N \\
+ & \left(\begin{array}{c}
-\frac{1}{2 \tau} x_{1} \kappa^{2} s^{2}-\frac{1}{\tau} x_{2} \kappa^{2} s \\
+\frac{1}{\tau} x_{3} \kappa+\frac{x_{1}}{\tau}
\end{array}\right) B_{1}(21) \\
+ & \left(\begin{array}{c}
\frac{1}{6 \tau} x_{1} \kappa^{2} \sigma s^{3}+\frac{1}{2 \tau} x_{2} \kappa^{2} \sigma s^{2} \\
-\frac{1}{\tau} x_{3} \kappa \sigma-\frac{x_{1} \sigma}{\tau} s+x_{4}
\end{array}\right) B_{2}
\end{aligned}
$$

where $x_{1}$ is a non-zero constant and $x_{2}, x_{3}, x_{4}$ are constants.

Proof. Let $\beta=\beta(s)$ be a unit speed timelike $W$-curve and $\delta=\delta\left(s_{\delta}\right)$ be its principal normal spherical image. If $\delta$ is a general helix, then for a spacelike vector $\Phi$, we may express

$$
g\left(T_{\delta}, \Phi\right)=\cos \theta
$$

where $\theta$ is a constant angle. The equation (22) is also congruent to

$$
g\left(\frac{\kappa T+\tau B_{1}}{\sqrt{\tau^{2}-\kappa^{2}}}, \Phi\right)=\cos \theta .
$$

The constant vector $\Phi$ according to $\left\{T, N, B_{1}, B_{2}\right\}$ is formed as

$$
\Phi=\varepsilon_{1} T+\varepsilon_{2} N+\varepsilon_{3} B_{1}+\varepsilon_{4} B_{2}
$$

Differentiating the expression (24) with respect to $s$, then we have the following system of ordinary differential equations

$$
\left\{\begin{array}{c}
\varepsilon_{1}^{\prime}+\varepsilon_{2} \kappa=0 \\
\varepsilon_{1} \kappa+\varepsilon_{2}^{\prime}-\varepsilon_{3} \tau=0 \\
\varepsilon_{2} \tau-\varepsilon_{4} \sigma+\varepsilon_{3}^{\prime}=0 \\
\varepsilon_{4}^{\prime}+\varepsilon_{3} \sigma=0
\end{array}\right.
$$

We know that $-\varepsilon_{1} \kappa+\varepsilon_{3} \tau=x_{1} \neq 0$ is a non-zero constant. Since the curve $\beta=\beta(s)$ is a $W$-curve, its curvature functions are constants. Then the solution of the system (25) can be obtained as

$$
\begin{aligned}
\varepsilon_{1}= & -\frac{1}{2} x_{1} \kappa s^{2}-x_{2} \kappa s+x_{3}, \\
\varepsilon_{2}= & x_{1} s+x_{2}, \\
\varepsilon_{3}= & -\frac{1}{2 \tau} x_{1} \kappa^{2} s^{2}-\frac{1}{\tau} x_{2} \kappa^{2} s \\
& +\frac{1}{\tau} x_{3} \kappa+\frac{x_{1}}{\tau}, \\
\varepsilon_{4}= & \frac{1}{6 \tau} x_{1} \kappa^{2} \sigma s^{3}+\frac{1}{2 \tau} x_{2} \kappa^{2} \sigma s^{2} \\
& -\frac{1}{\tau} x_{3} \kappa \sigma s-\frac{x_{1} \sigma}{\tau} s+x_{4} .
\end{aligned}
$$

3.2. The binormal spherical image of a timelike $W$-curve in $E_{1}^{4}$

In this section, we give the definition of the binormal spherical image for timelike $W$-curves in Minkowski space-time $E_{1}^{4}$.

Definition 3.6. Let $\beta=\beta(s)$ be a unit speed timelike $W$-curve in Minkowski space-time $E_{1}^{4}$. If we translate the binormal vector to the center 0 of the pseudohyperbolic space $\mathbb{H}_{0}^{3}$, we obtain a curve $\varphi=\varphi\left(s_{\varphi}\right)$. This curve is called the 
DEU FMD 22(64), 59-66, 2020

binormal spherical indicatrix or image of the curve $\beta$ in $E_{1}^{4}$.

Theorem 3.7. Let $\beta=\beta(s)$ be a unit speed timelike $W$-curve and $\varphi=\varphi\left(s_{\varphi}\right)$ be its binormal spherical image. Then,

i) $\varphi=\varphi\left(s_{\varphi}\right)$ is a spacelike curve.

ii) Frenet-Serret apparatus of the curve $\varphi,\left\{T_{\varphi}, N_{\varphi}, B_{1 \varphi}, B_{2 \varphi}, \kappa_{\varphi}, \tau_{\varphi}, \sigma_{\varphi}\right\}$ can be formed by the apparatus of the curve $\beta$.

iii) $\varphi=\varphi\left(s_{\varphi}\right)$ is also a helix lying on the pseudohyperbolic sphere $\mathbb{H}_{0}^{3}$ in $E_{1}^{4}$.

Proof. Let $\beta=\beta(s)$ be a unit speed timelike $W$-curve and $\varphi=\varphi\left(s_{\varphi}\right)$ be its binormal spherical image. It can be written as

$$
\varphi=B_{1}(s) .
$$

Differentiating the equation (27) with respect to $s$, then we obtain

$$
\varphi^{\prime}=\dot{\varphi} \frac{d s_{\varphi}}{d s}=-\tau N+\sigma B_{2} .
$$

Here, we shall denote differentiation according to $s$ by a dash, and differentiation according to $s_{\varphi}$ by a dot. Thus, we obtain the unit tangent vector of the binormal spherical image curve $\varphi$ as

$$
T_{\varphi}=\frac{-\tau N+\sigma B_{2}}{\sqrt{\tau^{2}+\sigma^{2}}}
$$

and

$$
\left\|\varphi^{\prime}\right\|=\frac{d s_{\varphi}}{d s}=\sqrt{\tau^{2}+\sigma^{2}} .
$$

The causal character of the binormal spherical image curve $\varphi$ is determined by the following inner product:

$$
g\left(\varphi^{\prime}, \varphi^{\prime}\right)=\tau^{2}+\sigma^{2}
$$

According to the expression (31), the binormal spherical image is a spacelike curve.

Considering the previous method and using the property of the curve to be $W$-curve, the following differentiations with respect to $s$ are formed:

$$
\begin{gathered}
\varphi^{\prime \prime}=-\tau \kappa T-\left(\tau^{2}+\sigma^{2}\right) B_{1}, \\
\varphi^{\prime \prime \prime}=\tau\left(\begin{array}{c}
\tau^{2}+\sigma^{2} \\
-\kappa^{2}
\end{array}\right) N-\sigma\left(\tau^{2}+\sigma^{2}\right) B_{2}, \\
\varphi^{(I V)}=\tau\left(\kappa\left(\tau^{2}+\sigma^{2}\right)-\kappa^{3}\right) T \\
\quad+\left(\left(\tau^{2}+\sigma^{2}\right)^{2}-\tau^{2} \kappa^{2}\right) B_{1} .
\end{gathered}
$$

By the expressions (2), then we get

$$
\begin{aligned}
& \left\|\varphi^{\prime}\right\|^{2} \varphi^{\prime \prime}-g\left(\varphi^{\prime}, \varphi^{\prime \prime}\right) \varphi^{\prime}=-\left(\tau^{2}+\right. \\
& \left.\sigma^{2}\right) \tau \kappa T-\left(\tau^{2}+\sigma^{2}\right)^{2} B_{1} .
\end{aligned}
$$

Then, we can get the principal normal vector of the binormal spherical image curve $\varphi$

$$
\begin{aligned}
N_{\varphi}= & -\frac{\kappa \tau}{\sqrt{\left|\left(\tau^{2}+\sigma^{2}\right)^{2}-(\tau \kappa)^{2}\right|}} T \\
& -\frac{\tau^{2}+\sigma^{2}}{\sqrt{\left|\left(\tau^{2}+\sigma^{2}\right)^{2}-(\tau \kappa)^{2}\right|}} B_{1},
\end{aligned}
$$

and the first curvature of the binormal spherical image curve $\varphi$ is as:

$$
\kappa_{\varphi}=\frac{\sqrt{\left|\left(\tau^{2}+\sigma^{2}\right)^{2}-(\tau \kappa)^{2}\right|}}{\tau^{2}+\sigma^{2}} .
$$

The vector product $X=T_{\varphi} \times N_{\varphi} \times \varphi^{\prime \prime \prime}$ is given by

$$
=-\frac{1}{\sqrt{\left|\left(\tau^{2}+\sigma^{2}\right)^{2}-(\tau \kappa)^{2}\right|\left(\tau^{2}+\sigma^{2}\right)}}\left(\begin{array}{c}
\left(\tau^{2}+\sigma^{2}\right) T \\
+(\kappa \tau) B_{1}
\end{array}\right) .
$$

Using the expression (36), then the trinormal (second binormal) vector field of the binormal spherical image curve $\varphi$ is obtained as

$$
\begin{aligned}
& B_{2 \varphi} \\
& =-\frac{\mu}{\kappa^{2} \tau \sigma \sqrt{\left|\left(\tau^{2}+\sigma^{2}\right)^{2}-(\tau \kappa)^{2}\right|}}\left(\begin{array}{c}
\left(\tau^{2}+\sigma^{2}\right) T \\
+(\kappa \tau) B_{1}
\end{array}\right) .
\end{aligned}
$$

Taking the norm of both sides of the equations (33) and (36), then we find the second curvature of the binormal spherical image curve $\varphi$

$$
\tau_{\varphi}=\frac{\kappa^{2} \tau \sigma}{\left(\tau^{2}+\sigma^{2}\right) \sqrt{\left(\tau^{2}+\sigma^{2}\right)^{2}-(\kappa \tau)^{2}}} .
$$


DEU FMD 22(64), 59-66, 2020

The binormal vector field of the the binormal spherical image curve $\varphi$ is expressed as

$$
B_{1 \varphi}=-\frac{\mu}{\sqrt{\tau^{2}-\kappa^{2}}}\left(\begin{array}{c}
\sigma N \\
+\tau B_{2}
\end{array}\right)
$$

Finally, using the equation (39), then the third curvature of the the binormal spherical image curve $\varphi$ is obtained by

$$
\sigma_{\varphi}=0
$$

Corollary 3.8. Frenet-Serret apparatus of the binormal spherical image $\varphi$ is an orthonormal frame of Minkowski space-time $E_{1}^{4}$.

Proof. It can be straightforwardly seen by using the equations (29), (34), (37), (39).

Corollary 3.9. Let $\beta=\beta(s)$ be a unit speed timelike $W$-curve and $\varphi=\varphi\left(s_{\varphi}\right)$ be its binormal spherical image. Then, the curve $\varphi$ is also a helix.

Proof. Let $\beta=\beta(s)$ be a unit speed timelike $W$-curve. We know that the curvature functions are constants. We know that the curvature functions of the binormal spherical image $\varphi\left(s_{\varphi}\right)$ are constants. Hence, the curve $\varphi\left(s_{\varphi}\right)$ becomes $W$-curve which is the special case of helix.

Theorem 3.10. Let $\beta=\beta(s)$ be a unit speed timelike $W$-curve and $\varphi=\varphi\left(s_{\varphi}\right)$ be its binormal spherical image. If $\varphi$ is a general helix, then its fixed direction $\Phi$ is composed

$$
\begin{aligned}
& \Phi=\left(\frac{1}{6 \tau} x_{1} \sigma^{2} \kappa s^{3}\right.+\frac{x_{2} \sigma^{2} \kappa s^{2}}{2 \tau} \\
&-\frac{1}{\tau} x_{3} \kappa \sigma \\
&\left.+\frac{1}{\tau} x_{1} \kappa \sigma+x_{4}\right) T \\
&+\left(-\frac{1}{2 \tau} x_{1} \sigma^{2} s^{2}-\frac{1}{\tau} x_{2} \sigma^{2} s\right. \\
&\left.+\frac{1}{\tau} x_{3} \sigma-\frac{x_{1}}{\tau}\right) N \\
&+\left(x_{1} s+x_{2}\right) B_{1} \\
&+\left(-\frac{1}{2} x_{1} \sigma s^{2}-x_{2} \sigma s+x_{3}\right) B_{2}
\end{aligned}
$$

where $x_{1}$ is a non-zero constant and $x_{2}, x_{3}, x_{4}$ are constants.
Proof. Let $\beta=\beta(s)$ be a unit speed timelike $W$-curve and $\varphi=\varphi\left(s_{\varphi}\right)$ be its binormal spherical indicatrix. If $\varphi$ is a general helix, then for a constant spacelike vector $\Phi$, we may express

$$
g\left(T_{\varphi}, \Phi\right)=\cos \theta
$$

where $\theta$ is a constant angle. The equation (28) is also congruent to

$$
g\left(\frac{-\tau N+\sigma B_{2}}{\sqrt{\tau^{2}+\sigma^{2}}}, \Phi\right)=\cos \theta
$$

The constant vector $\Phi$ according to $\left\{T, N, B_{1}, B_{2}\right\}$ is formed as

$$
\Phi=\varepsilon_{1} T+\varepsilon_{2} N+\varepsilon_{3} B_{1}+\varepsilon_{4} B_{2}
$$

Differentiating the expression (43) with respect to $s$, then we have the following system of ordinary differential equations

$$
\left\{\begin{array}{c}
\varepsilon_{1}^{\prime}+\varepsilon_{2} \kappa=0 \\
\varepsilon_{1} \kappa+\varepsilon_{2}^{\prime}-\varepsilon_{3} \tau=0 \\
\varepsilon_{2} \tau-\varepsilon_{4} \sigma+\varepsilon_{3}^{\prime}=0 \\
\varepsilon_{4}^{\prime}+\varepsilon_{3} \sigma=0
\end{array}\right.
$$

We know that $-\varepsilon_{2} \tau+\varepsilon_{4} \sigma=x_{1} \neq 0$ is a non-zero constant. Since the curve $\beta=\beta(s)$ is a $W$-curve, its curvature functions are constants. Then the solution of the system (44) can be obtained as

$$
\begin{gathered}
\varepsilon_{1}=\frac{1}{6 \tau} x_{1} \sigma^{2} \kappa s^{3}+ \\
+\frac{x_{2} \sigma^{2} \kappa s^{2}}{2 \tau}-\frac{1}{\tau} x_{3} \kappa \sigma \\
+\frac{1}{\tau} x_{1} \kappa \sigma+x_{4}, \\
\varepsilon_{2}=-\frac{1}{2 \tau} x_{1} \sigma^{2} s^{2}-\frac{1}{\tau} x_{2} \sigma^{2} s+\frac{1}{\tau} x_{3} \sigma \\
-\frac{x_{1}}{\tau}, \\
\varepsilon_{3}=x_{1} s+x_{2}, \\
\varepsilon_{4}=-\frac{1}{2} x_{1} \sigma s^{2}-x_{2} \sigma s+x_{3} .
\end{gathered}
$$

\section{Discussion and Conclusion}

In the present work, we extend spherical image concept to timelike $W$-curve in Minkowski space-time. We investigate principal normal and 
DEU FMD 22(64), 59-66, 2020

binormal spherical images of a timelike $W$-curve and observe that principal normal spherical curves are spacelike curves under certain conditions, and also binormal spherical images occur entirely as spacelike curves. Thereafter, we determine relations between Frenet-Serret invariants of the base curve and its spherical images.

\section{Acknowledgement}

The authors would like to thank anonymous reviewers for their valuable comments and suggestions to improve the quality of the paper.

\section{References}

[1] O'Neill, B. 1983. Semi-Riemannian Geometry with Applications to Relativity, Academic Press Inc. London.

[2] Milman, R.S., Parker, G.D. 1977. Elements of Differential Geometry, Prentice-Hall Inc. Englewood Cliffs, New Jersey.

[3] Walrave, J. 1995. Curves and Surfaces in Minkowski Space, Dissertation, K.U. Leuven, Fac. of Science, Leuven, $147 \mathrm{p}$.

[4] Yllmaz, S. 2001. Spherical Indicators of Curves and Characterizations of Some Special Curves in Four Dimensional Lorentzian Space $L^{4}$, Dissertation, Dokuz Eylül University, İzmir

[5] Yllmaz, S., Turgut, M. 2008. On the Differential Geometry of the Curves in Minkowski Space-Time I, International Journal of Contemporary Mathematical Sciences Volume 3, pp. 1343-1349.

[6] Yılmaz, S., Özyılmaz, E., Turgut, M. 2009. On the Differential Geometry of the Curves in Minkowsk Space-Time II, International Journal of
Contemporary Mathematical Sciences Volume 3, pp. 53-55.

[7] Yılmaz, S., Özyılmaz, E., Yaylı, Y., Turgut, M. 2010. Tangent and Trinormal Spherical Images of a TimeLike Curve on the Pseudohyperbolic Space, Proceedings of the Estonian Academy of Sciences, Volume 59 No 3, pp. 216-224.

[8] Bonnor, W.B. 1969. Null Curves in Minkowski Space-Time, Tensor, Volume 20, pp. 229-242.

[9] Petrovic-Torgasev, M., Sucurovic, E. 2002. WCurves in Minkowski Space-Time, Novi Sad Journal of Mathematics, Volume 32, No 2, pp. 55-65.

[10] Lopez, R. 2010. Differential Geometry of Curves and Surfaces in Lorentz-Minkowski Space, International Electronic Journal of Geometry, Volume 3, No 2, pp. 67-101.

[11] Ratcliffe, J.G. 2006. Foundations of Hyperbolic Manifolds, Springer Science-Business Media, LLC, New York, pp. 68-72.

[12] Monterde, J. 2007. Curves with Constant Curvature Ratios, Boletin de la Sociedad Matematica Mexicana, Volume 3, pp. 177-186.

[13] Öztürk, G., Arslan, K., Hacısalihoğlu H.H. 2008. A Characterization of CCR-Curves in $R^{m}$, Proceedings of the Estonian Academy of Sciences, Volume 57, pp. 217-224.

[14] İlarslan, K., Boyacıoğlu, Ö. 2007. Position Vectors of a Spacelike W-Curve in Minkowski 3-Space $E_{1}^{3}$, Volume 44, No 3, pp. 429-438.

[15] Camcl, C.., İlarslan, K., Sucurovic, E. 2003. On Pseudohyperbolic Curves in Minkowski SpaceTime, Turkish Journal of Mathematics, Volume 27, pp. 315-328.

[16] İlarslan, K., 2008. Boyacıoğlu, Ö., Position vectors of a timelike and a null helix in Minkowski 3-space, Chaos, Solitons and Fractals 38, 1383-1389.

[17] Kalkan Ö.B., 2016. Position vector of a W-curve in the 4D Galilean Space ${ }^{4}$, Facta Universitatis (NIS) Ser. Math. Inform., Vol. 31, No:2, 485-492. 